\title{
Bilateral total extraperitoneal inguinal hernia repair (TEP) has outcomes similar to those for unilateral TEP: population-based analysis of prospective data of 6,505 patients
}

\author{
Markus Gass • Laura Rosella • Vanessa Banz • \\ Daniel Candinas • Ulrich Güller
}

Received: 17 May 2011/Accepted: 20 October 2011/Published online: 24 November 2011

(C) Springer Science+Business Media, LLC 2011

\begin{abstract}
Background Whether bilateral total extraperitoneal (TEP) inguinal hernia repair is associated with worse outcomes than unilateral TEP continues to be a matter of debate. This study aimed to compare different outcomes of large cohorts of patients undergoing bilateral versus unilateral TEP.

Methods Based on prospective data of the Swiss Association of Laparoscopic and Thoracoscopic Surgery (SALTS), all patients undergoing elective unilateral or bilateral TEP from 1995 to 2006 were included in the study. The outcomes compared included conversion rates; intraoperative, surgical, and general postoperative complications; duration of operation; and length of hospital stay (LOS). Unadjusted and risk-adjusted multivariable analyses were performed.

Results Data for 6,505 patients undergoing unilateral $(n=3,457)$ and bilateral $(n=3,048)$ TEP were prospectively collected. The average age and the American Society
\end{abstract}

Presented as podium presentation at, the European Surgical Association, Annual Meeting, May 2010, Budapest, Hungary; the Swiss Surgical Society, Annual Meeting, June 2010, Interlaken, Switzerland; and the European Society for Surgical Research, Annual Meeting, June 2010, Geneva, Switzerland.

M. Gass · V. Banz · D. Candinas · U. Güller $(\bowtie)$

Department of Visceral Surgery and Medicine, University

Hospital Bern, 3010 Bern, Switzerland

e-mail: ulrich.guller@gmail.com

L. Rosella

Dalla Lana School of Public Health, University of Toronto,

Toronto, ON, Canada

U. Güller

Department of Oncology \& Hematology, Cantonal Hospital

St. Gallen, CH-9007, St. Gallen, Switzerland of Anesthesiologists (ASA) score were similar in the two groups. The patients undergoing bilateral TEP repair had a slightly increased rate of intraoperative complications (bilateral, $3.1 \%$ vs. unilateral, $1.9 \%$ ) and surgical postoperative complications (bilateral, $3.2 \%$ vs. unilateral, $2.3 \%$ ). The operation time was longer for bilateral TEP repair (86 vs. $67 \mathrm{~min}$ ). No significant differences in postoperative LOS, general postoperative complications, or conversion rates were found.

Conclusions This is the first population-based analysis in the literature to compare different outcomes in a prospective cohort of more than 6,500 patients undergoing bilateral versus unilateral TEP. Although the rates for intraoperative and surgical postoperative complications were slightly higher for the patients undergoing bilateral TEP repair, the absolute differences were small and of minor clinical relevance. Bilateral TEP repair is associated with a minimal increase in operating time and similar LOS, general postoperative complications, and conversion rates. Therefore, for patients with bilateral inguinal hernia, a simultaneous endoscopic approach represents an excellent therapeutic option.

Keywords Bilateral vs. unilateral inguinal hernia repair . Inguinal hernia - Population-based analysis - TEP - Total extraperitoneal inguinal hernia repair

Symptomatic inguinal hernia is a common surgical problem, and inguinal hernia repair is among the most frequently performed operations in general surgery [1, 2]. Indeed, more than 800,000 groin hernia repairs are performed in the United States each year [3]. Approximately $15 \%$ of all inguinal hernias are bilateral [4]. The debate continues as to whether bilateral hernias should be repaired simultaneously or sequentially $[5,6]$. 
The advantages of simultaneous repair chiefly include patient convenience and economic aspects because only one admission, one anesthesia, and one period of convalescence are required [7]. Nevertheless, because bilateral hernia repair is thought to result in a higher incidence of postoperative complications and a higher recurrence rate, some surgeons advocate a two-stage procedure.

Several endoscopic techniques for inguinal hernia repair have been developed over the past two decades. Currently, two endoscopic procedures - total extraperitoneal (TEP) and transabdominal preperitoneal (TAPP) inguinal hernia repair-are the most commonly performed [8-10]. Although the fully extraperitoneal approach, performed without entering the abdominal cavity, is perceived by some surgeons as an advantage of TEP over TAPP repair, no major differences have been found $[11,12]$.

In the setting of open inguinal hernia repair, the question whether surgery for a bilateral hernia should be simultaneous or sequential has been addressed extensively, and several authors have failed to show any difference in terms of most postoperative complications, return to daily activities, or long-time recurrence rate [13, 14]. However, the question whether bilateral TEP repair is associated with poorer outcomes than unilateral TEP repair remains unanswered because the available literature is scarce. Therefore, the current analysis aimed to compare relevant outcomes in a large prospective cohorts of patients between unilateral and bilateral TEP.

\section{Materials and methods}

This analysis is based on data from the Swiss Association of Laparoscopic and Thoracoscopic Surgery (SALTS) database, a prospective database of consecutive patients undergoing laparoscopic procedures in Switzerland.

\section{Inclusion and exclusion criteria}

The current study enrolled all patients 18 years of age or older who underwent elective primary unilateral or bilateral TEP for inguinal hernia from 1995 to 2006. Patients who had recurrent uni- or bilateral hernia were excluded. All data were prospectively collected and entered in a centralized database (Qualicare; Qualidoc, Liebefeld-Bern, Switzerland) by a data manager who worked independently of the authors of this study. Values missing from data sheets were obtained by the data manager. Baseline demographic data were extracted, as well as data regarding the following clinical outcomes: conversion rate, intraoperative complications, postoperative surgical complications (e.g., wound infection, wound hematoma), general postoperative complications (e.g., pneumonia, urinary tract infection, pulmonary embolism), length of operation, and postoperative length of hospital stay (LOS).

Statistical analysis

All statistical computations were performed by an experienced Ph.D. statistician (L.R.). Outcomes were summarized using means/median and standard deviations (for continuous variables) or proportions (for categorical variables). For continuous outcomes, the mean differences between uni- and bilateral hernia were tested using a two-sample $t$-test. Categorical variables and rates were tested for differences using a chisquare test. Multivariable-adjusted analysis was performed on the following outcomes: intraoperative complications, postoperative surgical complications, general postoperative complications, conversion rates, postoperative LOS, and operating times. Complications and conversion outcomes were modeled using logistic regression. For intra- and postoperative complications, the event of the univariate and multivariable analysis for each outcome was defined as the presence of one or more complications. The duration of surgery was dichotomized such that the outcome was defined as being in the upper quartile (above the 75th percentile) of the distribution.

The postoperative LOS was modeled using a linear regression analysis. All multivariable models were adjusted for age, gender, and American Society of Anesthesiologists (ASA) score. A $P$ value of 0.05 was considered statistically significant. All statistical tests were two-sided. All statistical analyses were performed using SAS version 9.1 (SAS Institute, Cary, NC, USA).

\section{Results}

From 1995 to 2006, the data of 6,505 patients undergoing elective unilateral $(n=3,457)$ or bilateral $(n=3,048)$ TEP repair for inguinal hernia were prospectively collected and entered into the database. Most of the patients (95.4\%) were men $(94.6 \%$ in the unilateral group and $96.3 \%$ in the bilateral group). The median age at the time of the procedure was 55 years (range, 18-89 years). The median ASA score was 1 (range, 1-4). The two groups were similar in terms of average age (unilateral, 53.6 years vs. bilateral, 54.6 years) and ASA score (unilateral, 1.4 vs. bilateral, 1.5).

The patients undergoing bilateral TEP repair had slightly increased rates for intraoperative complications (bilateral, $3.1 \%$ vs. unilateral, $1.9 \% ; P=0.002$ ) and surgical postoperative complications (bilateral, $3.2 \%$ vs. unilateral, $2.3 \%$; $P=0.026)$. The duration of surgery was longer for the patients undergoing bilateral TEP $(86 \mathrm{~min})$ than for those undergoing unilateral TEP $(67 \mathrm{~min})(P<0.001)$. No significant differences between the bilateral and unilateral TEP groups were observed in terms of postoperative LOS (2.5 vs. 
Table 1 Unadjusted comparison of outcomes for patients undergoing unilateral versus bilateral total extraperitoneal (TEP) inguinal hernia repair

\begin{tabular}{llllll}
\hline Variable & $\begin{array}{l}\text { Unilateral } \\
\text { TEP }(n=3,457) \\
n(\%)\end{array}$ & SD & $\begin{array}{l}\text { Bilateral } \\
\text { TEP }(n=3,048) \\
n(\%)\end{array}$ & $\begin{array}{l}\text { SD value needed } \\
\text { to treat }\end{array}$ \\
\hline Intraoperative complications & $65(1.9)$ & - & $94(3.1)$ & - & 0.002 \\
Surgical postop complications & $78(2.3)$ & - & $96(3.2)$ & - & 0.026 \\
General postop complications & $25(0.7)$ & - & $29(1.0)$ & - & 0.151 \\
Conversion to open procedure & $33(1.0)$ & - & $32(1.1)$ & - & 0.699 \\
Postop length of stay (days) & 2.3 & 6.4 & 2.5 & 7.0 & 0.196 \\
Duration of operation (min) & 67 & 31.0 & 86 & 36.0 & $<0.001$ \\
\hline
\end{tabular}

$S D$ standard deviation; postop postoperative; $N A$ not applicable

2.3 days; $P=0.196)$, general postoperative complications (1.0 vs. $0.7 \% ; P=0.151$ ), or conversion rates (1.1 vs. $1.0 \%$; $P=0.699)$. The respective unadjusted and risk-adjusted comparisons of outcomes are displayed in Tables 1 and 2.

\section{Discussion}

To our knowledge, this is the first population-based analysis to compare outcomes in a prospective and consecutive cohort of more than 6,500 patients between bilateral and unilateral TEP. The current investigation provides compelling evidence that bilateral TEP repair is associated with a minimal increase in operating time, intraoperative complications, and surgical postoperative complications and is characterized by similarity to unilateral TEP in terms of hospital stay, incidence of general postoperative complications, and conversion rates.

Although, observed differences with respect to intraoperative complications and surgical postoperative complications attained statistical significance in the current investigation, the absolute differences were very small and of minor clinical relevance. For instance, the absolute difference in terms of intraoperative complications between unilateral (1.9\%) and bilateral $(3.1 \%)$ TEP repair was only $1.2 \%$, which is of negligible clinical relevance. Similarly, the absolute difference in surgical postoperative complications between unilateral $(2.3 \%)$ and bilateral $(3.2 \%)$ TEP repair was only $0.9 \%$.

It must be emphasized that these very small differences become statistically significant due to the very large sample size and the associated high power of the study [15-17]. Indeed, the number needed to treat are 83 and 111 for intraoperative and surgical postoperative complications in our investigation, respectively (Table 1). This means that if 83 patients undergo bilateral endoscopic hernia repair, there is only 1 additional intraoperative complication compared to a series of patients undergoing unilateral hernia repair. Similarly, if 111 patients undergo bilateral
Table 2 Risk-adjusted comparison of outcomes for patients undergoing unilateral versus bilateral total extraperitoneal (TEP) inguinal hernia repair

\begin{tabular}{|c|c|c|c|c|}
\hline Variable & OR & LCL & UCL & $P$ value \\
\hline Intraoperative complications & 1.66 & 1.20 & 2.29 & 0.002 \\
\hline $\begin{array}{l}\text { Surgical postoperative } \\
\text { complications }\end{array}$ & 1.38 & 1.02 & 1.86 & 0.0395 \\
\hline $\begin{array}{l}\text { General postoperative } \\
\text { complications }\end{array}$ & 1.30 & 0.76 & 2.23 & 0.3396 \\
\hline Conversion to open procedure & 1.07 & 0.66 & 1.75 & 0.7832 \\
\hline \multirow{2}{*}{$\begin{array}{l}\text { Duration of operation in upper } \\
\text { quartile of the operation }\end{array}$} & 2.77 & 2.46 & 3.11 & $<0.0001$ \\
\hline & Beta & SE & & $P$ value \\
\hline Postoperative length of stay & 0.2079 & 0.1665 & & 0.2118 \\
\hline
\end{tabular}

OR odds ratio; $L C L$ lower $95 \%$ confidence limit; $U C L$ upper $95 \%$ confidence limit; $S E$ standard error

endoscopic hernia repair, there is only 1 additional surgical postoperative complication compared to a series of patients undergoing unilateral hernia repair.

No significant differences in general postoperative complications, postoperative LOS, or conversion rate were observed. The unilateral surgery time was shorter than the bilateral surgery time by only approximately $20 \mathrm{~min}$.

These data correspond to findings from a much smaller study reported by Lau et al. [18], in which 103 patients undergoing bilateral TEP for inguinal hernia were prospectively investigated during 3 years and compared with an age-matched cohort of patients who underwent unilateral TEP during the same period. The duration of surgery in the study of Lau et al. [18] (unilateral, $64 \mathrm{~min}$ vs. bilateral, $97 \mathrm{~min}$ ) was comparable with that in our study. The recovery time, mean LOS (unilateral, 1.5 days vs. bilateral, 1.7 days), and incidence of postoperative general complications and postoperative surgical complications did not differ significantly between the two groups. Our current analysis of more than 6,500 patients confirms the findings of this much smaller study. 
An investigation in Sweden included 380 patients with unilateral inguinal hernia and 64 patients with bilateral inguinal hernia who underwent surgery using either TEP or TAPP repair [19]. No differences were observed in terms of complications or time to full recovery between unilateral and bilateral inguinal hernia repair. The LOS was slightly prolonged in the bilateral group. Nevertheless, $41 \%$ of these patients were able to leave the hospital on the same day as the operation.

In the current analysis, no significant difference in postoperative LOS was found between the two patient groups. The postoperative LOS reflects both the patient's perception of pain and the time needed for recovery from general anesthesia. Because there is no need for further trocar placement and because the additional dissection of the preperitoneal space for bilateral hernia is only marginal, the systemic impact on the patient's physiology is of minor relevance. This is demonstrated by data from a series of 373 hernias in 250 patients reported by Ahmad and Schuricht [20]. Of these 250 patients, 127 received unilateral repair and 123 had bilateral repair with the TEP technique. The conversion rate was zero in both groups (i.e., it was possible to complete all the operations endoscopically). No differences between the two groups were noted in terms of patient-reported perception of pain, postoperative narcotic use, level of activity, or time to resumption of work.

Although, some of the end points investigated in the study by Ahmad and Schuricht [20] differ from those explored in our series and although no complications were reported, this much smaller study largely accords with our findings, with no difference in the average time to patient recovery or the average LOS between the two groups of patients. Moreover, the slight increase in operating time for the patients undergoing bilateral endoscopic hernia repair was of minor clinical relevance and well tolerated by the patients.

A very recent retrospective, single-surgeon study compared the outcomes of patients undergoing simultaneous bilateral versus unilateral TEP repair [21]. Over a period of 6 years, 879 patients (involving 112 bilateral and 767 unilateral cases) underwent inguinal hernia repair. Although the operating time was slightly longer for the bilateral group, the authors found no differences in hospital stay or postoperative complications.

\section{Study strengths and limitations}

Our study is most limited by a lack of long-term data. For instance, we were unable to assess the incidence of hernia recurrence, long-term pain, or degree of patient satisfaction with bilateral versus unilateral TEP repair. Additionally, although the SALTS dataset provides information on whether patients experienced complications or not, the grading of the complications cannot be ascertained. Finally, the current analysis investigated only one method for inguinal hernia repair. Further research comparing the outcomes of different inguinal hernia repair methods (e.g., TAPP vs. TEP) in large prospective cohorts would be of great interest.

The current study has a number of strengths. First, the large sample size conferred a high degree of statistical power for detecting clinically relevant differences. Second, the data, all gathered prospectively and queried for incomplete values, were very complete. Third and finally, although we examined only patients treated in Switzerland, we believe the results of this population-based study can be extrapolated to other countries with high standards of laparoscopic surgical technique.

This study represents the first population-based analysis to compare different outcomes in a large prospective cohort of patients undergoing unilateral versus bilateral TEP. Although, the intraoperative complications and the surgical postoperative complications were statistically higher among the patients undergoing bilateral TEP repair (due to the large sample size and high statistical power), the absolute differences were of minor clinical relevance. The slight increase in complications was counterbalanced by the avoidance of all the potential problems, inconveniences, and costs of two hospital stays and two operations, each time with the patient under general anesthesia. Bilateral TEP is associated with a minimal increase in operating time, intraoperative and postoperative surgical complications, and is characterized by similar length of hospital stay, rate of general postoperative complications, and conversion rates compared with unilateral TEP. For patients with bilateral inguinal hernia, an endoscopic approach using the TEP technique represents an excellent therapeutic option that can be performed with outcomes similar to those seen with unilateral endoscopic hernia repair.

Acknowledgment The authors thank Jonathan McCall for his careful reading of the manuscript and many valuable suggestions. This study was supported by grants from Johnson \& Johnson and Covidien.

Disclosures Markus Gass, Laura Rosella, Vanessa Banz, Daniel Candinas, and Ulrich Güller have no conflicts of interest or financial ties to disclose.

\section{References}

1. McCormack K, Wake BL, Fraser C, Vale L, Perez J, Grant A (2005) Transabdominal preperitoneal (TAPP) versus totally extraperitoneal (TEP) laparoscopic techniques for inguinal hernia repair: a systematic review. Hernia 9:109-114

2. Kurzer M, Kark A, Hussain T (2007) Inguinal hernia repair. J Perioper Pract 17:318-330 
3. Rutkow IM (2003) Demographic and socioeconomic aspects of hernia repair in the United States in 2003. Surg Clin North Am 83:1045-1051 v-vi

4. Fischer S, Cassivi S, Paul A, Troidl H (1999) Evidence-based medicine and special aspects in bilateral inguinal hernia repair. Hernia 3:89-95

5. Pawanindra L, Philips P, Chander J, Ramteke VK (2010) Is unilateral laparoscopic TEP inguinal hernia repair a job half done? The case for bilateral repair. Surg Endosc 24:1737-1745

6. Wauschkuhn CA, Schwarz J, Boekeler U, Bittner R (2010) Laparoscopic inguinal hernia repair: gold standard in bilateral hernia repair? Results of more than 2,800 patients in comparison to literature. Surg Endosc 24:3026-3030

7. Pfeffer F, Riediger H, Kufner Lein R, Hopt UT (2008) Repair of bilateral inguinal hernias: Sequential or simultaneous? Zentralbl Chir 133:446-451 discussion 452

8. Dulucq JL, Wintringer P, Mahajna A (2009) Laparoscopic totally extraperitoneal inguinal hernia repair: lessons learned from 3,100 hernia repairs over 15 years. Surg Endosc 23:482-486

9. Dulucq JL (1992) Treatment of inguinal hernia by insertion of a subperitoneal patch under preperitoneoscopy. Chirurgie 118:83-85

10. Arregui ME, Davis CJ, Yucel O, Nagan RF (1992) Laparoscopic mesh repair of inguinal hernia using a preperitoneal approach: a preliminary report. Surg Laparosc Endosc 2:53-58

11. Leibl BJ, Jager C, Kraft B, Kraft K, Schwarz J, Ulrich M, Bittner $\mathrm{R}$ (2005) Laparoscopic hernia repair: TAPP or/and TEP? Langenbecks Arch Surg 390:77-82
12. Krahenbuhl L, Schafer M, Buchler MW (1997) Laparoscopic transperitoneal inguinal hernia operation (TAPP). Chirurg 68:977-985

13. Miller AR, van Heerden JA, Naessens JM, O'Brien PC (1991) Simultaneous bilateral hernia repair: a case against conventional wisdom. Ann Surg 213:272-276

14. Serpell JW, Johnson CD, Jarrett PE (1990) A prospective study of bilateral inguinal hernia repair. Ann R Coll Surg Engl 72:299-303

15. Guller $U$ (2008) Caveats in the interpretation of the surgical literature. Br J Surg 95:541-546

16. Guller U, DeLong ER (2004) Interpreting statistics in medical literature: a vade mecum for surgeons. J Am Coll Surg 198:441-458

17. Guller U, Oertli D (2005) Sample size matters: a guide for surgeons. World J Surg 29:601-605

18. Lau H, Patil NG, Yuen WK (2003) A comparative outcome analysis of bilateral versus unilateral endoscopic extraperitoneal inguinal hernioplastics. J Laparoendosc Adv Surg Tech A 13:153-157

19. Kald A, Domeij E, Landin S, Wiren M, Anderberg B (2000) Laparoscopic hernia repair in patients with bilateral groin hernias. Eur J Surg 166:210-212

20. Ahmad SA, Schuricht AL (1997) A comparison of patient recovery following unilateral and bilateral endoscopic preperitoneal herniorrhaphy. JSLS 1:231-235

21. Choi YY, Hur KY (2011) Simultaneous laparoscopic totally extraperitoneal repair of bilateral inguinal hernia: review of 1 surgeon experiences. Surg Laparosc Endosc Percutan Tech 21:264-266 